\title{
Caracterização da micobiota e detecção de aflatoxina M1 em suplementos alimentares para recém-nascidos para uso em unidade neonatal
}

\author{
Victor Moebus Farias', Beatriz Clarissa dos Santos Ferreira e Pereira1 ${ }^{1}$, Leonardo de Assunção Pinto², \\ Luiz Antonio Moura Keller ${ }^{3}$, Robson M. Franco ${ }^{3}$
}

\begin{abstract}
Resumo
Nos dias atuais, o uso de suplementos alimentares se faz cada vez mais presente devido a mudanças nos hábitos alimentares. Sendo assim, se tornou cada vez mais necessário a inclusão de suplementos alimentares na dieta de crianças, em especial os recém-nascidos. Considerando a fragilidade da população alvo, é importante realizar estudos para a avaliação dos possíveis perigos microbiológicos e toxicológicos aos quais os consumidores estão expostos. As análises microbiológicas foram realizadas utilizando meios específicos para enumeração fúngica e as concentrações de micotoxinas detectadas através de fluorimetria associada com métodos imuno enzimáticos. As análises revelaram valores médios (UFC/mL): fungos filamentosos e xerofílicos $8,14 \times 10^{2}$ e 2,34 x $10^{3}$, respectivamente. Os valores de contagem obtidos nesse estudo são um reflexo de falhas durante o processamento do produto, escolha da matéria prima e manipulação durante a sua reconstituição. Porém, as amostras analisadas apresentaram valores de contagem dentro do preconizado pela legislação estando assim em conformidade e seguras para o consumo.
\end{abstract}

Palavras-chave: Proteínas do soro do leite; Contagem fúngica; Aflatoxina M1.

\section{Introdução}

O leite humano é o único alimento ingerido em quantidades suficientes por recém-nascidos apresentando um elevado valor nutricional, energético e imunológico. O leite materno é importante par o crescimento e desenvolvimento do recémnascido, além de auxiliar a formação do sistema imune, do sistema nervoso e facilitar o desenvolvimento emocional e cognitivo (WHO, 2018).

A prática do aleitamento materno teve uma redução a partir da década de 40 até a década de 70 . Essa tendência começou a ser atenuada em meados da década de 80 , quando tiveram início os programas de incentivo ao aleitamento materno (WHO, 2009). Com a redução dessa prática, tem sido necessário a utilização de suplementos alimentares para suprir a necessidade nutricional dos recém-nascidos.

As fórmulas utilizadas na suplementação de recém-nascidos podem ser definidas como produto, em forma líquida ou em pó, utilizado quando indicado, para lactentes sadios a partir do sexto mês de vida até doze meses de idade incompletos (11 meses e 29 dias) e para crianças de primeira infância sadias, constituindose o principal elemento líquido de uma dieta progressivamente diversificada (BRASIL, 2011a). A legislação brasileira ainda prevê os padrões de identidade e qualidade das fórmulas infantis (BRASIL, 2011a), padrão de qualidade microbiológico (BRASIL, 2001), assim como os limites máximos de micotoxinas no produto final (BRASIL, 2011b). No entanto, a legislação brasileira não apresenta limites para presença de fungos em fórmulas infantis.

Os suplementos alimentares, podem estar associados a diversos tipos de contaminação microbiológicas provenientes dos ingredientes utilizados em suas formulações, como é o caso da utilização de proteína do soro de leite como principal fonte de proteína, que pode estar associada a diversos microrganismos, incluindo as bactérias do grupo dos coliformes, Escherichia coli e Staphylococcus aureus. A presença de fungos, em geral o gênero Aspergillus, como contaminantes nessas amostras estão associadas aos componentes de origem vegetal, frequentemente relacionado à produção de toxinas (GERMANO; GERMANO, 2011).

Micotoxinas são metabólitos secundários de fungos filamentosos que se desenvolvem em diversos produtos de origem agrícola, ainda na lavoura e também no período de estocagem. As micotoxinas em formulas infantis, estão associadas aos gêneros Aspergillus, Fusarium e Penicillium, originárias tanto de produtos de origem vegetal quanto animal e, apesar de não apresentarem quadros agudos, a ingestão prolongada está relacionada com infecções crônicas (FORSYTHE, 2002).

O gênero Aspergillus é mundialmente conhecido como produtor de toxinas, principalmente em regiões de climas mais quentes, onde a estocagem de grãos não é feita de forma adequada, favorecendo a proliferação do fungo e a produção de aflatoxina do tipo B. Esses grãos, quando utilizados na fabricação de rações para animais, acabam por contaminar os animais. A aflatoxina B1 é então metabolizada e excretada no leite como aflatoxina M1 (AFM1), capaz de produzir efeitos tóxicos agudos e carcinogênicos em diversas espécies animais. Há a possibilidade da AFM1 se ligar de forma irreversível a caseína do leite, resistindo a pasteurização e outros processos para a produção de derivados lácteos (GERMANO; GERMANO, 2011).

\footnotetext{
*Aluno de mestrado do curso de Higiene Veterinária e Tecnologia de Produtos de Origem Animal, Universidade Federal Fluminense, Niterói, Brasil. Bolsista da Coordenação de Aperfeiçoamento de Pessoal de Nível Superior (CAPES).

**Aluno de graduação do curso de Farmácia, Universidade Federal Fluminense, Niterói, Brasil.

*** Professor de Medicina Veterinária, Universidade Federal Fluminense, Niterói, Brasil. Rua Vital Brazil Filho 64 - cep: $24230-340$ - Vital Brazil - Niterói -

RJ. E-mail: victormoebus@id.uff.br
} 
Tendo em vista os componentes usualmente utilizados no preparo das fórmulas infantis, podemos classificar o grupo das aflatoxinas como mais importantes de se avaliar no produto final. Uma vez que o principal componente das fórmulas infantis é a proteína do soro do leite, a aflatoxina M1 é principal toxina envolvida nos quadros de intoxicação por micotoxinas. Os limites máximos para essa toxina em leite cru estão descritos no Codex alimentarius (FAO, 2014). Alegislação brasileira apresenta valores similares quanto ao leite cru, além de apresentar limites permitidos para leite em pó, enquadrando assim as fórmulas infantis $(0,5 \mu \mathrm{g} / \mathrm{L})$. Devido a utilização de componentes de origem vegetal na formulação, ainda estão descritos os valores para outros tipos de aflatoxinas (BRASIL, 2011b).

Estudos que avaliem e monitorem essas matrizes, quanto aos riscos e contaminantes, atestam não apenas o padrão de identidade e qualidade dos produtos, mas também, subsidiam a comunidade de dados importantes quando a produtos de amplo consumo. Considerando a fragilidade da população alvo, o presente estudo tem como objetivo estudar e avaliar a qualidade microbiológica dos produtos oferecidos em unidades neonatais a fim de conhecê-los e avalia-los dentro dos limites de seguridade para a população alvo.

\section{Material e Métodos}

As amostras de fórmulas infantis para a primeira infância foram fornecidas pelo lactário do Hospital Universitário Antônio Pedro (HUAP). Foram analisadas 36 amostras, sendo um total de seis lotes de duas marcas reconhecidas no mercado, avaliadas em três formas: fórmula na forma sólida, coletada no momento da abertura da embalagem primária (T1); fórmula na forma líquida reconstituída no momento da reconstituição da amostra (T2) e a fórmula na forma líquida reconstituída após 24 horas de manutenção da refrigeração (T3). As amostras foram avaliadas em duplicatas.

As amostras avaliadas foram coletadas assepticamente em tubos estéreis. As amostras sólidas (T1) foram coletadas em tubos de centrífuga estéreis que foram abertos no momento da coleta e as demais amostras (T2 e T3) em mamadeiras esterilizadas autoclavadas utilizadas pelo lactário para oferta de fórmulas infantis, bem como os demais utensílios utilizados no manuseio e preparação dos produtos.

As amostras foram preparadas seguindo os protocolos operacionais de manuseio do HUAP para poder avaliar corretamente a qualidade dos produtos oferecidos aos pacientes. A amostra T3 foi mantida refrigerada na própria unidade de lactário para avaliação do comportamento da amostra nas condições de armazenamento da unidade.

Por serem as amostras T1 um produto em pó, não foi necessário transportá-las sob refrigeração. As amostras após a ressuspensão foram transportadas sob refrigeração imediatamente para o laboratório onde foram realizadas as análises, visando manter as características das amostras. Amostras T3 de cada lote foram transportadas nas mesmas condições de refrigeração após 24 horas.

Para a contagem de fungos, foi realizada a diluição decimal seriada em placas, com inoculação de alíquotas de $0,1 \mathrm{~mL}$ de cada uma das diluições em ágar Dicloran Rosa de Bengala Cloranfenicol (DRBC) para estimar o total de fungos (ABARCA et al., 1994). As placas foram incubadas a $25^{\circ} \mathrm{C}$ por sete dias. Todas as análises foram realizadas com duplicatas analíticas.

A amostras foram extraídas utilizando o método de extração baseado no QuEChERS modificado, seguindo metodologia descrita no manual de métodos oficiais de análises da AOAC (2007). A triagem das amostras foi realizada utilizando kits imunoenzimáticos comerciais para AFM1 (Aflatest ${ }^{\circledR}$, Vicam, Watertown, MA, EUA), seguindo instruções do fabricante. Para quantificação e análise foi realizada avaliação em fluorímetro VICAM® Series-4EX (Watertown, MA, EUA). Os padrões de AFM1 (5 mg) foram adquiridos com a Sigma (St. Louis, MO, USA). A solução estoque $\left(50 \mu \mathrm{g} \mathrm{mL}^{-1}\right)$ e as soluções de trabalho $\left(2 \mu \mathrm{g} \mathrm{mL}^{-1}\right)$ foram preparados em metanol e suas concentrações confirmadas por absorção de luz UV utilizando um espectofotômetro Shimadzu UV-1201 (Kyoto, Japan) (AOAC 2007), estocadas em frascos âmbar a $-10^{\circ} \mathrm{C}$ por um período de três meses. Os limites de detecção (LOD) e quantificação (LOQ) foram encontrados pela adição, decrescente, de concentrações da solução padrão e submetidas a extração e quantificação até a menor concentração detectável (LOD) e a menor concentração quantificável (LOQ), sob condições adequadas de repetitividade $(n=5, R S D<15 \%)$. Os limites de detecção e quantificação encontrados foram $0,013 \mu \mathrm{gg}^{-1}$ e $0,055 \mu \mathrm{g} \mathrm{kg}^{-1}$, respectivamente.

\section{Resultados e Discussão}

Após a realização das análises descritas, foram obtidos os valores de contagem total de fungos encontrado apresentados na tabela 1 e as frequências absolutas e percentual de cepas fúngicas isoladas apresentadas na tabela 2 :

Tabela 1: Contagem total (UFC/g) para, fungos filamentosos (DRBC) em formulas infantis

\begin{tabular}{cc}
\hline Amostras & DRBC \\
\hline T1 & $1,39 \times 10^{3} \pm 1,16 \times 10^{3} \mathrm{a}$ \\
T2 & $5,17 \times 10^{2} \pm 3,50 \times 10^{2} \mathrm{~b}$ \\
T3 & $5,33 \times 10^{2} \pm 4,93 \times 10^{2} \mathrm{c}$ \\
\hline
\end{tabular}

*LOD: $\leq 1,0 \times 101 \mathrm{UFC/g;} \mathrm{**a,} \mathrm{b} \mathrm{e} \mathrm{c:} \mathrm{Médias} \mathrm{com} \mathrm{mesma} \mathrm{letra} \mathrm{nas} \mathrm{colunas}$ são equivalentes, de acordo com o teste Tukey $(P \leq 0,05)$.

Tabela 2: Frequências absolutas e percentual de cepas fúngicas dos gêneros isolados nas amostras de suplementos para recém-nascidos

$\begin{array}{ccc}\text { Gênero fúngico } & \begin{array}{c}\text { Número absoluto } \\ \text { em UFC }\end{array} & \text { Frequência (\%) }\end{array}$

Carga Fúngica Total das Amostras Avaliadas

\begin{tabular}{ccc} 
Aspergillus sp. & 42 & 53,16 \\
Penicillium sp. & 19 & 24,05 \\
Cladosporium sp. & 6 & 7,59 \\
Mucor sp. & 6 & 7,60 \\
Fusarium sp. & 6 & 7,60 \\
Total & 79 & 100,0 \\
\hline
\end{tabular}

No primeiro ponto analisado (T1), foi observado um valor médio de $1,39 \times 10^{3} \mathrm{UFC} / g$ para fungos filamentosos. Uma 
vez que as embalagens foram abertas no momento da coleta, a contaminação inicial observada pode ser oriunda da matéria prima e manipulação durante o processamento tecnológico. Santos et al. (2014) atribuíram a contaminação tanto a qualidade inferior das matérias primas que compõe o produto final, bem como falhas no processamento do produto.

Fungos são conhecidos como contaminantes ambientais. Por isso, o local aonde as amostras são manipuladas também são pontos críticos a serem analisados quanto a contaminação fúngica. Devido a facilidade de dispersão desses microorganismos e protocolos de limpeza ineficazes, fungos podem ser encontrados em sistemas fechados de ventilação aderidos aos filtros utilizados, sendo indicadores biológicos com um limite máximo de $7,50 \times 10^{2} \mathrm{UFC} / \mathrm{m}^{3}$ (BRASIL, 2003).

Martins-Diniz et al. (2005) avaliaram a presença de fungos em ambientes hospitalares e obtiveram valores médios de contagem de $3,33 \times 10^{3} \mathrm{UFC} / \mathrm{m}^{3}$ em unidade de terapia intensiva, valores muito acima do preconizado pela legislação brasileira. Em outros estudos foram observados valores elevados de contaminação fúngica em ambientes hospitalares, considerandose principalmente a bandeja do sistema de ar condicionado como principal fonte de proliferação microbiana e os gêneros Aspergillus spp. e Penicillium spp. como contaminante fúngicos de maior frequência. Confirmando assim a contaminação devido ao controle ineficaz da sanitização do sistema de ar condicionado (MOBIN; SALMITO, 2006). Corroborando os achados apresentados na tabela 2.

Quando analisados o segundo tempo das amostras (T2), observou-se declínio significativo da contagem após a adição de água quente para a ressuspensão das amostras, atingindo valores de 5,17 × $10^{2} \mathrm{UFC} / \mathrm{mL}$ para fungos filamentosos.

Foi perceptível que a adição de água quente nas amostras, para reconstituição das amostras, foi suficiente para causar a inativação celular, reduzindo os valores de contagem fúngica, até que a amostra se encontre em condições adequadas para o crescimento de indivíduos menos afetados Groot et al. (2019) corroboraram os resultados observados anteriormente referente a utilização da aplicação de aquecimento a $60^{\circ} \mathrm{C}$ para inativar fungos do gênero Penicillium.

\section{Referências}

ABARCA, M.L.; BRAGULAT, MR.; CASTELLA, G.; CABANES, P.J. Ochratoxin A production by strains of Aspergillus niger var. niger. Applied and Environmental Microbiology. v.60, p 2650-2652, 1994.

ALVITO, P. C.; SIZOO, E. A.; ALMEIDA, C. M. M.; van EGMOND, H. P. Occurrence of Aflatoxins and Ochratoxin A in Baby Foods in Portugal. Food Analitycal Methods, v. 3, p. 22-30. 2010.

AOAC. Association of Official Analytical Chemists. Official Methods of Analysis 19 ed. Gaithersburgh, Maryland: Association of Official Analytical Chemists International 2007.

BRASIL. Ministério da Saúde. Agência Nacional de Vigilância Sanitária (ANVISA). Resolução da diretoria colegiada- RDC $n^{\circ}$ 12, de 2 de janeiro de 2001. Aprova o regulamento técnico sobre padrões microbiológicos para alimentos, em anexo. Diário Oficial [da] União, Brasília, DF, n. 7, p. 45, 10 jan. 2001. Seção 1.
Após o período de armazenagem, o ponto T3 apresentou um aumento em relação a contagem anterior, sendo observados contagens obtidas de $5,33 \times 10^{2} \mathrm{UFC} / \mathrm{mL}$ para fungos filamentosos. $O$ aumento nas contagens observadas no terceiro ponto do estudo são diretamente relacionados a contaminação ambiental durante a manipulação, que introduziu novas células viáveis que não sofreram injúrias celulares pela adição de água aquecida, possibilitando o crescimento durante o período de 24 horas de armazenagem.

Após a análise de micotoxinas nas amostras, foi possível observar que todas as amostras apresentaram concentrações abaixo do limite de detecção da técnica $(\leq 0,013 \mathrm{mg} / \mathrm{kg})$.

A ausência de micotoxinas nas amostras avaliadas no presente trabalho são sugestivos do aumento gradativo na precaução e controle de micotoxinas a nível global conforme descrito por Alvito et al. (2010) e Tonon, Savi e Scussel (2018).

O cuidado com a AFM1 deve partir da qualidade inicial da amostra uma vez que a toxina é termo resistente, resistindo a temperaturas elevadas como demostrado por lha et al. (2013) no qual foram encontrados em concentrações de AFM1 em uma faixa de 20 - 760 $\mathrm{ng} / \mathrm{kg}$ em $100 \%$ das amostras de leite UHT e em pó avaliadas.

\section{Conclusões}

Apesar de não existirem padrões específicos para contagem fúngica na legislação brasileira, os produtos analisados se encontram em conformidade com as normativas internacionais vigentes. Sendo considerados seguros para o consumo, porém se reforça a necessidade de consumo imediato após ressuspensão a fim de evitar desenvolvimento fúngico indesejado.

Apesar dos produtos analisados estarem em conformidade, as contaminações apontadas nesse estudo apontam a necessidade de maiores esforços quanto ao controle do processo de fabricação dos produtos, sejam eles a qualidade da matéria prima utilizada ou os processos de limpeza dos equipamentos empregados pela indústria. Além, da necessidade do treinamento periódico dos profissionais de unidades de lactário que irão manipular esses produtos e da revisão dos protocolos de higienização dos utensílios utilizados.

BRASIL. Ministério da Saúde. Agência Nacional de Vigilância Sanitária (ANVISA). Resolução-RE no 9, de 16 de janeiro de 2003. Determina a publicação de orientação técnica elaborada por grupo técnico assessor, sobre padrões referenciais de qualidade do ar interior, em ambientes climatizados artificialmente de uso público e coletivo. Diário Oficial [da] União, Brasília, DF, n. 114, p. 35, 20 jan. 2003. Seção 1.

BRASIL. Ministério da Saúde. Agência Nacional de Vigilância Sanitária (ANVISA). Resolução da diretoria colegiada- RDC $n^{\circ} 44$, de 19 de setembro de 2011. Regulamento técnico para fórmulas infantis de seguimento para lactentes e crianças de primeira infância. Diário Oficial [da] União, Brasília, DF, n. 182, p. 92, 21 set. 2011, a. Seção 1.

BRASIL. Ministério da saúde. Agência Nacional de Vigilância Sanitária. Resolução da diretoria colegiada- RDC $n^{\circ} 7$, de 18 de fevereiro de 2011. Dispõe sobre limites máximos tolerados (LMT) para micotoxinas em alimentos. Diário Oficial [da] União, Brasília, DF, n. 7, p. 72, 22 fev. 2011b. Seção 1. 
FAO/WHO Food and Agriculture Organization of the United Nations/World Health Organization. General standard for contaminants and toxins in food and feed CXS 193-1995. 2014. $65 \mathrm{p}$.

FORSYTHE, S. J. Microbiologia da Segurança Alimentar. Porto Alegre: Artmed, 2002. 422p.

GERMANO, P. M. L.; GERMANO, M. I. S. Higiene e Vigilância Sanitária de Alimentos. 4. ed. São Paulo: Manole, 2011. 1034 p.

GROOT, M. N.; ABEE, T.; VEEN, H. B. Inactivation of conidia from three Penicillium spp. isolated from fruit juices by conventional and alternative mild preservation technologies and disinfection treatments. Food Microbiology, v. 81, p. 108-114. 2019.

IHA, M. H.; BARBOSA, C. B.; OKADA, I. A.; TRUCKSESS, M. W. Aflatoxin M1 in milk and distribution and stability of aflatoxin M1 during production and storage of yoghurt and cheese. Food Control, v. 29, p. 1-6. 2013.

MARTINS-DINIZ, J. N.; SILVA, R. A. M.; MIRANDA, E. T.; MENDES-GIANNINNI, M. J. S. Monitoramento de fungos anemófilos e de leveduras em unidade hospitalar. Revista Saúde Pública, v. 39, n. 3, p. 398-405. 2005.
MOBIN, M.; SALMITO, M. A. Microbiota fúngica dos condicionadores de ar nas unidades de terapia intensiva de Teresina, PI. Revista da Sociedade Brasileira de Medicina Tropical, v. 36, n. 6, p. 556- 559. 2006.

SANTOS, J. S.; OKANO, W.; ARRAIS, B. C. D.; COSTABEBER, I. H.; SANTANA, E. H. W. Aflatoxin M1 in Dairy Products and Acid Lactic Bacteria as Biocontrol Agent in Milk. Uniciências, v. 18, n. 1, p. 51-56. 2014.

TONON, K. M.; SAVI, G. D.; SCUSSEL, V. M. Application of a LC-MS/MS method for multimycotoxin analysis in infant formula and milkbased products for young children commercialized in Southern Brazil. Journal of Environmental Science and Health, Part B, v. 0, n. 0, p. 1-7. 2018.

WORLD HEALTH ORGANIZATION, UNITED NATIONS CHILDREN'S FUNDANTION. WHO. Baby-friendly hospital initiative. Revised, Updated and Expanded for Integrated Care. Geneva, 2009.

WORLD HEALTH ORGANIZATION, UNITED NATIONS CHILDREN'S FUNDANTION. WHO. Protecting, promoting and supporting breastfeeding in facilities providing maternity and newborn services: the revised Baby-friendly hospital initiative. Geneva, 2018. 